\title{
Clickable Galactose Analogs for Imaging Glycans in Developing Zebrafish
}

\author{
Jessica L. Daughtry ${ }^{1}$, Wendy Cao ${ }^{1}$, Johnny $\mathrm{Ye}^{1}$, and Jeremy M. Baskin ${ }^{1 *}$
}

\begin{abstract}
1 Department of Chemistry and Chemical Biology and Weill Institute for Cell and Molecular Biology, Cornell University, 461 Weill Hall, Ithaca, New York, USA 14853

* Correspondence to Jeremy Baskin: jeremy.baskin@cornell.edu
\end{abstract}

\begin{abstract}
Galactose is one of only nine monosaccharide precursors used to build complex glycans in vertebrates. Defects in galactose metabolism cause galactosemia and lysosomal storage diseases, and the ability to visualize metabolic flux through these pathways would help to understand mechanisms underlying disease pathogenesis. Bioorthogonal metabolic reporters are widely used tools to image glycan biosynthesis, but to date, no galactose analogs have capitalized on this strategy. We demonstrate that the galactose salvage pathway is remarkably intolerant of unnatural galactose and galactose-1-phosphate analogs. Subtle modifications to uridine diphosphate galactose (UDP-Gal), the universal donor for galactosyltransferases, however, yielded effective metabolic probes for labeling glycans in vivo. We applied 6-alkynyl UDP-Gal, followed by click chemistry tagging, to visualize glycosylation during zebrafish development, revealing a striking accumulation into glycan-rich ridges within the organism's enveloping layer. UDP-Gal analogs represent a new class of glycan metabolic probes for revealing physiological and pathological changes in glycosylation in vivo.
\end{abstract}




\section{INTRODUCTION}

Glycans are the most structurally complex of all the natural biopolymers, and the chemical diversity of protein glycosylation dwarfs that of other posttranslational modifications. Yet, in the cells of higher eukaryotes, glycans are built from a small set of only nine monosaccharide precursors. Among these privileged building blocks, galactose (Gal) plays myriad important roles in the cell. The historical importance of Gal in glycobiology and human health is underpinned by its discovery ${ }^{1}$ and structural determination ${ }^{2}$ by Pasteur and Fischer, respectively, and its later elucidation as a key constituent of the $\mathrm{ABO}$ blood group antigens $\mathrm{s}^{3-5}$. Gal is now appreciated to be a ubiquitous component of nearly all major forms of glycans, including $\mathrm{N}$ - and O-linked glycoproteins and several types of glycolipids ${ }^{6}$.

Unsurprisingly, defects in Gal metabolism can cause severe diseases. Heritable loss-of-function mutations in genes encoding enzymes that assimilate and catabolize dietary Gal via the Leloir pathway result in galactosemia, which features a buildup of toxic Gal-derived metabolites ${ }^{7}$. Severe galactosemia causes infant mortality, and milder forms can permit life, though with serious developmental, physical, and cognitive disabilities even under a highly lactose-restricted dietary regime ${ }^{8-10}$. Defects in the breakdown of Galcontaining glycans cause several lysosomal storage diseases that also have severe health consequences ${ }^{11}$. For example, Krabbe disease results from loss of a single galactosidase responsible for hydrolysis of galactosylceramide lipids, leading to demyelination, neurodegeneration, and infant mortality ${ }^{12,13}$.

Though the genetic and biochemical defects underlying these disorders of galactose metabolism are established, the diseases are currently incurable. This clinical challenge 
arises in part because glycosylation is not directly encoded in the genome. Instead, it is the product of a complex web of metabolic and membrane trafficking reactions, where alterations to a single player (i.e., a metabolic enzyme or a transporter) can have complex and unpredictable effects on overall changes in pathway flux and the identities and abundances of the resultant glycan structures and metabolic intermediates. A key set of tools that has emerged over the past two decades to unravel the complexity of glycan biosynthesis are metabolic reporters that enable the direct visualization of flux through these pathways.

Metabolic oligosaccharide engineering involves the delivery to cells of synthetic monosaccharide precursors that hijack glycan biosynthetic pathways and are incorporated into glycan structures ${ }^{14-16}$. Following the metabolic labeling step, these reporters can be subsequently tagged via a bioorthogonal click chemistry reaction ${ }^{17}$, which enables their visualization by fluorescence microscopy or enrichment by affinity chromatography for glycomic or glycoproteomic analysis. Sialic acids were the first glycan constituents to be labeled in this manner, using analogs of a biosynthetic precursor, $N$-acetylmannosamine ${ }^{18-}$ 23. Subsequently, several of the other monosaccharide constituents of vertebrate glycans were targeted by this strategy, including $N$-acetylgalactosamine ${ }^{24}, N$-acetylglucosamine ${ }^{25}$, fucose $\mathrm{e}^{26,27}$, xylose $^{28}$, and, most recently, glucose ${ }^{29}$. Importantly, metabolic labeling followed by bioorthogonal tagging is a viable strategy for imaging changes in glycosylation in vivo in animal models ${ }^{30-35}$, where changes in glycosylation can report on changes in development and disease progression.

Gal represents one of the few remaining monosaccharides used for vertebrate glycan biosynthesis that has not yet been targeted for imaging using metabolic reporters. 
Critically, tools for monitoring changes in Gal incorporation into glycans could aid in understanding changes in flux in glycan biosynthesis, trafficking, and degradation in diseases of Gal metabolism. Here, we describe the design, synthesis, and validation of unnatural Gal analogs as glycan metabolic reporters and their application to visualize glycosylation occurring during zebrafish embryonic and larval development. Our studies identify certain privileged Gal analogs as a new class of metabolic probes for glycosylation and suggest future applications to image changes in glycan biosynthesis that accompany both physiological and pathological changes in animal models of disease.

\section{RESULTS AND DISCUSSION}

To understand the substrate tolerance of the Gal salvage pathway, we prepared a panel of peracetylated Gal analogs in which a hydroxyl group was replaced with an azide or terminal alkyne (Figs. 1A and S1 and Scheme S1). These sugars were designed to be cell-permeable; following deacylation by cytosolic esterases, they could enter the Gal salvage (i.e., Leloir) pathway, which converts Gal to Gal-1-phosphate and then to uridine diphosphate Gal (UDP-Gal), the universal donor for galactosyltransferases (GalTs) that mediate addition of Gal to glycoproteins and glycolipids (Fig. 1A). None of the six analogs afforded metabolic labeling of cell-surface glycans, as assessed by flow cytometry following biotin tagging via $\mathrm{Cu}$-catalyzed azide-alkyne cycloaddition (CuAAC) with either alkyne-biotin or azido-biotin conjugate and streptavidin-fluorophore staining (Fig. S1). Notably, the phosphorylation of Gal at the 1 position, representing the first committed step in the Gal salvage pathway, is catalyzed by galactokinase (GALK), whose crystal structure 
reveals several key hydrogen bonds to the hydroxyl groups of Gal that may explain the very limited tolerance of GALK to unnatural substrates ${ }^{36-38}$.

To probe the tolerance of later Gal salvage pathway steps to unnatural substrates, we bypassed the restrictive GALK-catalyzed step by preparing a 6-deoxyazido analog of Gal-1-phosphate, the product of the GALK reaction. We designed this analog to be cell permeable by peracetylation of hydroxyl groups and protection of the phosphate with hydrophobic and esterase-sensitive $S$-acetyl thioethyl (SATE) groups (Fig. S2 and Scheme $\mathrm{S} 2)^{39,40}$. We confirmed the use of the SATE strategy for enabling cell permeability by using it to cage a known azide-modified metabolic reporter analog of $N$-acetylglucosamine-1phosphate ${ }^{41}$ as a positive control (Fig. S2). Interestingly, the SATE-protected 6-azido-Gal1-phosphate analog exhibited no detectable metabolic labeling of cell-surface glycans (Fig. $\mathrm{S} 2)$.

One possible conclusion from these studies is that even very small modifications to Gal that would permit its tagging using bioorthogonal chemistry do not enable metabolic labeling via the salvage pathway, in contrast to the majority of other reported monosaccharides. The other possibility was that these precursors were being successfully converted to the corresponding UDP-Gal analog but that these nucleotide sugars were either not recognized by transporters for import into the Golgi apparatus or were not substrates of GalTs.

To distinguish between these possibilities, we synthesized a 6-deoxyazido analog of UDP-Gal (UDP-6AzGal, Fig. 1A) and evaluated whether it could be accepted as a GalT substrate. For these studies, we used ceramide galactosyltransferase (CGT), which couples UDP-Gal and ceramides to form a $\beta$-galactosylceramide (GalCer) products that can be 
detected by liquid chromatography-mass spectrometry (LC-MS). We performed an in vitro glycosylation reaction by treating lysates from HEK $293 \mathrm{TN}$ cells overexpressing CGT with a non-natural, 17-carbon ceramide donor and UDP-6AzGal (Fig. 2A). To enhance MS detection, we carried out a subsequent CuAAC tagging reaction with an alkynyl quaternary ammonium reagent (Alk-QA $)^{42,43}$. Gratifyingly, we observed formation of the expected QA-modified GalCer product, confirming that UDP-6AzGal could indeed act as a GalT substrate (Fig. 2B).

We next strove to apply UDP-6AzGal as a metabolic substrate for visualizing glycosylation in live cells. Because of their negative charges, nucleotide sugars are not cellpermeable, and thus we turned to zebrafish embryos, which can be easily microinjected and serve as a powerful vertebrate system for imaging the biosynthesis of several types of glycans during embryonic and larval development using the bioorthogonal metabolic reporter strategy $28,30,44-46$.

In these studies, we microinjected either UDP-6AzGal or an analogous alkynyl analog, UDP-6AlGal (Scheme S3), whose acetylene tag represents a slightly smaller perturbation than the azido group, into the yolk of zebrafish embryos at the one-cell stage, allowing for permeation of the unnatural sugar into the cell. Following 24 hours of development, we observed minimal toxicity and developmental defects at the injected doses with UDP-6AlGal and moderate toxicity with UDP-6AzGal. We thus proceeded with UDP-6AlGal for all future studies in zebrafish.

First, we generated lysates at the 24 hour-post fertilization (hpf) timepoint, and the metabolically labeled glycoproteins were tagged with azido-biotin via CuAAC, followed by Western blot with streptavidin-HRP. We observed substantial labeling of glycoproteins 
(Fig. 3A and B). To determine the types of glycans being labeled, we took advantage of chemical differences between $\mathrm{O}$ - and $\mathrm{N}$-linked glycans, which can be selectively removed either chemically or enzymatically, respectively.

To assess the extent of UDP-6AlGal labeling within O-glycans, the post-CuAAC lysate was transferred to a PVDF membrane following SDS-PAGE, and O-glycans were removed by $\beta$-elimination with sodium hydroxide. Under these conditions, we observed no loss of UDP-6AlGal-derived signal (Fig. 3A, C). As important controls, lysates from HeLa cells metabolically labeled with peracetylated $N$-(4-pentynoyl)mannosamine (Ac $\left.{ }_{4} \mathrm{ManNAl}\right)$ or $\mathrm{N}$-(4-pentynoyl)galactosamine ( $\left.\mathrm{Ac}_{4} \mathrm{GalNAl}\right)$, both of which result in a substantial level of incorporation into O-linked glycans ${ }^{21,22,47}$, led to a $\beta$-eliminationdependent loss of signal using this assay (Fig. S3). Thus, we conclude that only a negligible fraction of UDP-6AlGal signal derives from all forms of O-linked glycoproteins.

To assess the extent of UDP-6AlGal incorporation into N-linked glycans, we performed glycosidase digestion of metabolically labeled lysates with peptide Nglycosidase F (PNGase F), which cleaves N-glycans from the protein backbone. Following digestion, we performed CuAAC tagging with azido-biotin and Western blot analysis as above and observed a substantial $(\sim 50 \%)$ decrease in overall signal in the PNGase Fdigested samples (Fig. 3B, C). Again, we used lysates from HeLa cells metabolically labeled with $\mathrm{Ac}_{4} \mathrm{ManNAl}$ or $\mathrm{Ac}_{4} \mathrm{GalNAl}$ as important controls for this assay, observing the expected decrease in the samples labeled with $\mathrm{Ac}_{4} \mathrm{ManNAl}$ (due to its incorporation into sialic acids, which appear in both $\mathrm{N}$ - and O-linked glycans ${ }^{21,22}$ ) but not $\mathrm{Ac}_{4} \mathrm{GalNAl}$ (due to its incorporation into O-linked glycans ${ }^{47}$ ) (Fig. S3). 
Having characterized the nature of glycoprotein labeling by UDP-6AlGal in vivo, we applied this probe to image changes in glycosylation in vivo during zebrafish development. Zebrafish embryos were microinjected with UDP-6AlGal and a tracer, rhodamine-dextran, and click chemistry tagging and imaging of glycans on their enveloping layer was performed at different timepoints within the first five days of development, i.e., up to $120 \mathrm{hpf}$. For the click chemistry-mediated tagging, to ensure the highest signal-to-background, we used a two-step procedure involving a live embryocompatible CuAAC reaction ${ }^{48}$ with an azido-biotin reagent followed by streptavidin-Alexa Fluor 488 labeling.

Excitingly, we detected UDP-6AlGal-derived fluorescence as early as $12 \mathrm{hpf}$, corresponding to early segmentation (Fig. 4A). The labeling was observed throughout the enveloping layer, and the extent of labeling increased up to $24 \mathrm{hpf}$ and persisted through $120 \mathrm{hpf}$ (Fig. 4A). Importantly, minimal fluorescence was observed in negative control embryos injected with rhodamine-dextran only and similarly labeled via CuAAC (Fig. 4B). The use of a UDP-Gal analog was required to observe labeling, as microinjection of an unprotected alkynyl Gal-1-phosphate analog (8, Scheme S3) also resulted in no detectable labeling, further supporting the lack of tolerance of the Leloir pathway to unnatural modifications.

A close examination of the enveloping layer in UDP-6AlGal-labeled animals revealed strong fluorescence from ridged structures whose morphology changes over the course of embryonic and early larval development (Fig. 4C). Interestingly, this type of glycan labeling pattern of the epithelium ${ }^{30,45}$ is reminiscent of "ridge-mazes" previously observed on the skin of a variety of fish species ${ }^{49-51}$. These ornate ridged structures, which 
are species-specific, dramatically increase the surface area of the skin and are thought to provide protection against pathogens, osmotic shocks, and shear force ${ }^{49,52}$. Critically, they are rich in mucous substances, including membrane-associated and secreted glycans ${ }^{49,51,52}$. The appearance of strong UDP-6AlGal-derived labeling in these epidermal structures in zebrafish larvae suggests that they are particularly metabolically active areas for de novo glycoprotein synthesis at these developmental stages, which coincide with the beginning of swimming behavior ${ }^{53}$.

\section{CONCLUSION}

We have performed a detailed investigation of the ability of unnatural Gal analogs to serve as metabolic reporters for visualizing glycosylation in vivo. Using in vitro biochemical and cellular assays, we reveal that the Gal salvage pathway is notably intolerant of modified Gal or Gal-1-phosphate analogs, in contrast to several other monosaccharide salvage pathways. By contrast, a bioorthogonal analog of UDP-Gal, the universal donor for galactosylation, was an effective galactosyltransferase substrate and metabolic label for tagging glycans following its microinjection into zebrafish embryos. Intriguingly, the bulk of metabolic labeling afforded by UDP-6AlGal was found to be in $\mathrm{N}$-linked glycoproteins, and detailed examinations of the glycoproteins and glycan structures labeled by this sugar, as well as the glycosyltransferases that utilize it as a substrate, represent interesting future directions. Our in vivo imaging studies of UDP6AlGal to visualize glycosylation patterns in developing zebrafish suggest the utility of this metabolic probe to examine changes in glycosylation accompanying perturbations that model congenital human diseases of glycosylation in this organism. 


\section{ACKNOWLEDGMENTS}

J.M.B. acknowledges support from the Arnold and Mabel Beckman Foundation (Beckman Young Investigator Award) and the Alfred P. Sloan Foundation (Sloan Research Fellowship). This work made use of the Cornell University NMR Facility, which is supported in part by the NSF (CHE-1531632). We thank Joe Fetcho and Brian Miller for assistance with zebrafish experiments, Robyn Meech for generously providing a CGT plasmid, Kelley Moremen and Jeong Yeh Yang for assistance with in vitro galactosylation reactions, the Emr and Fromme labs for reagents and equipment, and members of the Baskin laboratory for helpful discussions.

\section{REFERENCES}

(1) Pasteur, L. Note Sur Le Sucre de Lait. In Comptes rendus de l'Académie des

Sciences, Volume 42; Académie des sciences (France): Paris, 1856; pp 347-351.

(2) Fischer, E.; Morrell, R. S. Ueber Die Configuration Der Rhamnose Und Galactose. Berichte der Dtsch. Chem. Gesellschaft 1894, 27 (1), 382-394. https://doi.org/10.1002/cber.18940270177.

(3) Landsteiner, K. Zur Kenntnis Der Antifermativen, Lytischen Und Agglutinierenden Wirkungendes Blutserums Und Der Lymphe. Zentbl. Bakt. Parasitkde (Abt.) 1900, 27, 357-363.

(4) Watkins, W. Biochemistry and Genetics of the ABO, Lewis, and P Blood Group Systems. Adv. Hum. Genet. 1980, 10, 1-136,379-385.

(5) Watkins, W. M. The ABO Blood Group System: Historical Background. Transfus. 
Med. 2001, 11 (4), 243-265. https://doi.org/10.1046/j.1365-3148.2001.00321.x.

(6) Essentials of Glycobiology, 3rd editio.; Varki, A., Cummings, R. D., Esko, J. D., Eds.; Cold Spring Harbor Laboratory Press: Cold Spring Harbor (NY), 2017.

(7) Holden, H. M.; Rayment, I.; Thoden, J. B. Structure and Function of Enzymes of the Leloir Pathway for Galactose Metabolism. J. Biol. Chem. 2003, 278 (45), 43885-43888. https://doi.org/10.1074/jbc.R300025200.

(8) Demirbas, D.; Coelho, A. I.; Rubio-Gozalbo, M. E.; Berry, G. T. Hereditary Galactosemia. Metabolism. 2018, 83, 188-196. https://doi.org/10.1016/j.metabol.2018.01.025.

(9) Timson, D. J. The Molecular Basis of Galactosemia - Past, Present and Future. Gene 2016, 589 (2), 133-141. https://doi.org/10.1016/j.gene.2015.06.077.

(10) Coelho, A. I.; Rubio-Gozalbo, M. E.; Vicente, J. B.; Rivera, I. Sweet and Sour: An Update on Classic Galactosemia. J. Inherit. Metab. Dis. 2017, 40 (3), 325-342. https://doi.org/10.1007/s10545-017-0029-3.

(11) Parenti, G.; Andria, G.; Ballabio, A. Lysosomal Storage Diseases: From Pathophysiology to Therapy. Annu. Rev. Med. 2015, 66 (1), 471-486. https://doi.org/10.1146/annurev-med-122313-085916.

(12) Wenger, D. A.; Rafi, M. A.; Luzi, P. Krabbe Disease: One Hundred Years from the Bedside to the Bench to the Bedside. J. Neurosci. Res. 2016, 94 (11), 982-989. https://doi.org/10.1002/jnr.23743.

(13) Graziano, A. C. E.; Cardile, V. History, Genetic, and Recent Advances on Krabbe Disease. Gene 2015, 555 (1), 2-13. https://doi.org/10.1016/j.gene.2014.09.046.

(14) Dube, D. H.; Bertozzi, C. R. Metabolic Oligosaccharide Engineering as a Tool for 
Glycobiology. Curr. Opin. Chem. Biol. 2003, 7 (5), 616-625.

https://doi.org/10.1016/j.cbpa.2003.08.006.

(15) Campbell, C. T.; Sampathkumar, S. G.; Yarema, K. J. Metabolic Oligosaccharide Engineering: Perspectives, Applications, and Future Directions. Mol. Biosyst. 2007, 3 (3), 187-194. https://doi.org/10.1039/b614939c.

(16) Sminia, T. J.; Zuilhof, H.; Wennekes, T. Getting a Grip on Glycans: A Current Overview of the Metabolic Oligosaccharide Engineering Toolbox. Carbohydr. Res. 2016, 435, 121-141. https://doi.org/10.1016/j.carres.2016.09.007.

(17) Patterson, D. M.; Nazarova, L. A.; Prescher, J. A. Finding the Right (Bioorthogonal) Chemistry. ACS Chem. Biol. 2014, 9 (3), 592-605. https://doi.org/10.1021/cb400828a.

(18) Kayser, H.; Zeitler, R.; Kannicht, C.; Grunow, D.; Nuck, R.; Reutter, W. Biosynthesis of a Nonphysiological Sialic Acid in Different Rat Organs, Using NPropanoyl-D-Hexosamines as Precursors. J. Biol. Chem. 1992, 267 (24), 1693416938.

(19) Mahal, L. K.; Yarema, K. J.; Bertozzi, C. R. Engineering Chemical Reactivity on Cell Surfaces through Oligosaccharide Biosynthesis. Science 1997, 276 (5315), 1125-1128. https://doi.org/10.1126/science.276.5315.1125.

(20) Saxon, E.; Bertozzi, C. R. Cell Surface Engineering by a Modified Staudinger Reaction. Science 2000, 287 (5460), 2007-2010. https://doi.org/10.1126/science.287.5460.2007.

(21) Chang, P. V; Chen, X.; Smyrniotis, C.; Xenakis, A.; Hu, T.; Bertozzi, C. R.; Wu, P. Metabolic Labeling of Sialic Acids in Living Animals with Alkynyl Sugars. 
Angew. Chemie Int. Ed. 2009, 48 (22), 4030-4033.

https://doi.org/10.1002/anie.200806319.

(22) Hsu, T.-L.; Hanson, S. R.; Kishikawa, K.; Wang, S.-K.; Sawa, M.; Wong, C.-H. Alkynyl Sugar Analogs for the Labeling and Visualization of Glycoconjugates in Cells. Proc. Natl. Acad. Sci. U. S. A. 2007, 104 (8), 2614-2619.

https://doi.org/10.1073/pnas.0611307104.

(23) Horstkorte, R.; Reutter, W. Metabolic Glycoengineering with N-Acyl Side Chain Modified Mannosamines. Angew. Chemie 2016, 128 (33), 9632-9665. https://doi.org/10.1002/ange.201601123.

(24) Hang, H. C.; Yu, C.; Kato, D. L.; Bertozzi, C. R. A Metabolic Labeling Approach toward Proteomic Analysis of Mucin-Type O-Linked Glycosylation. Proc. Natl. Acad. Sci. U.S.A 2003, 100 (25), 14846-14851.

https://doi.org/10.1073/pnas.2335201100.

(25) Vocadlo, D. J.; Hang, H. C.; Kim, E.-J.; Hanover, J. A.; Bertozzi, C. R. A Chemical Approach for Identifying O-GlcNAc-Modified Proteins in Cells. Proc. Natl. Acad. Sci. U. S. A. 2003, 100 (16), 9116-9121. https://doi.org/10.1073/pnas.1632821100.

(26) Sawa, M.; Hsu, T.-L.; Itoh, T.; Sugiyama, M.; Hanson, S. R.; Vogt, P. K.; Wong, C.-H. Glycoproteomic Probes for Fluorescent Imaging of Fucosylated Glycans in Vivo. Proc. Natl. Acad. Sci. U. S. A. 2006, 103 (33), 12371-12376. https://doi.org/10.1073/pnas.0605418103.

(27) Rabuka, D.; Hubbard, S. C.; Laughlin, S. T.; Argade, S. P.; Bertozzi, C. R. A Chemical Reporter Strategy to Probe Glycoprotein Fucosylation. J. Am. Chem. 
Soc. 2006, 128 (37), 12078-12079. https://doi.org/10.1021/ja064619y.

(28) Beahm, B. J.; Dehnert, K. W.; Derr, N. L.; Kuhn, J.; Eberhart, J. K.; Spillmann, D.; Amacher, S. L.; Bertozzi, C. R. A Visualizable Chain-Terminating Inhibitor of Glycosaminoglycan Biosynthesis in Developing Zebrafish. Angew. Chem. Int. Ed. Engl. 2014, 53 (13), 3347-3352. https://doi.org/10.1002/anie.201310569.

(29) Darabedian, N.; Gao, J.; Chuh, K. N.; Woo, C. M.; Pratt, M. R. The Metabolic Chemical Reporter 6-Azido-6-Deoxy-Glucose Further Reveals the Substrate Promiscuity of O-GlcNAc Transferase and Catalyzes the Discovery of Intracellular Protein Modification by O-Glucose. J. Am. Chem. Soc. 2018, 140 (23), 7092-7100. https://doi.org/10.1021/jacs.7b13488.

(30) Laughlin, S. T.; Baskin, J. M.; Amacher, S. L.; Bertozzi, C. R. In Vivo Imaging of Membrane-Associated Glycans in Developing Zebrafish. Science 2008, 320 (5876), 664-667. https://doi.org/10.1126/science.1155106.

(31) Chang, P. V; Prescher, J. A.; Sletten, E. M.; Baskin, J. M.; Miller, I. A.; Agard, N. J.; Lo, A.; Bertozzi, C. R. Copper-Free Click Chemistry in Living Animals. Proc. Natl. Acad. Sci. U.S.A 2010, 107 (5), 1821-1826. https://doi.org/10.1073/pnas.0911116107.

(32) Xie, R.; Dong, L.; Du, Y.; Zhu, Y.; Hua, R.; Zhang, C.; Chen, X. In Vivo Metabolic Labeling of Sialoglycans in the Mouse Brain by Using a LiposomeAssisted Bioorthogonal Reporter Strategy. Proc. Natl. Acad. Sci. 2016, 113 (19), 5173-5178. https://doi.org/10.1073/pnas.1516524113.

(33) Laughlin, S. T.; Bertozzi, C. R. In Vivo Imaging of Caenorhabditis Elegans Glycans. ACS Chem. Biol. 2009, 4 (12), 1068-1072. 
https://doi.org/10.1021/cb900254y.

(34) Rong, J.; Han, J.; Dong, L.; Tan, Y.; Yang, H.; Feng, L.; Wang, Q. W.; Meng, R.;

Zhao, J.; Wang, S. Q.; et al. Glycan Imaging in Intact Rat Hearts and

Glycoproteomic Analysis Reveal the Upregulation of Sialylation during Cardiac

Hypertrophy. J. Am. Chem. Soc. 2014, 136 (50), 17468-17476.

https://doi.org/10.1021/ja508484c.

(35) Prescher, J. A.; Dube, D. H.; Bertozzi, C. R. Chemical Remodelling of Cell

Surfaces in Living Animals. Nature 2004, 430 (7002), 873-877.

https://doi.org/10.1038/nature02791.

(36) McAuley, M.; Kristiansson, H.; Huang, M.; Pey, A. L.; Timson, D. J.

Galactokinase Promiscuity: A Question of Flexibility? Biochem. Soc. Trans. 2016, 44 (1), 116-122. https://doi.org/10.1042/bst20150188.

(37) Timson, D. J.; Reece, R. J. Sugar Recognition by Human Galactokinase. BMC

Biochem. 2003, 4, 1-8. https://doi.org/10.1186/1471-2091-4-16.

(38) Thoden, J. B.; Timson, D. J.; Reece, R. J.; Holden, H. M. Molecular Structure of Human Galactokinase: Implications for Type II Galactosemia. J. Biol. Chem.

2005, 280 (10), 9662-9670. https://doi.org/10.1074/jbc.M412916200.

(39) Yu, S.-H.; Boyce, M.; Wands, A. M.; Bond, M. R.; Bertozzi, C. R.; Kohler, J. J. Metabolic Labeling Enables Selective Photocrosslinking of O-GlcNAc-Modified Proteins to Their Binding Partners. Proc. Natl. Acad. Sci. 2012, 109 (13), 4834 4839. https://doi.org/10.1073/pnas.1114356109.

(40) Lefebvre, I.; Perigaud, C.; Pompon, A.; Aubertin, A.-M.; Girardet, J.-L.; Kirn, A.; Gosselin, G.; Imbach, J.-L. Mononucleoside Phosphotriester Derivatives with S- 
Acyl-2-Thioethyl Bioreversible Phosphate-Protecting Groups: Intracellular Delivery of 3'-Azido-2',3'-Dideoxythymidine 5'-Monophosphate. J. Med. Chem. 1995, 38 (20), 3941-3950. https://doi.org/10.1021/jm00020a007.

(41) Tan, H. Y.; Eskandari, R.; Shen, D.; Zhu, Y.; Liu, T. W.; Willems, L. I.; Alteen, M. G.; Madden, Z.; Vocadlo, D. J. Direct One-Step Fluorescent Labeling of OGlcNAc-Modified Proteins in Live Cells Using Metabolic Intermediates. $J$. Am. Chem. Soc. 2018, 140 (45), 15300-15308. https://doi.org/10.1021/jacs.8b08260.

(42) Bumpus, T. W.; Baskin, J. M. Clickable Substrate Mimics Enable Imaging of Phospholipase D Activity. ACS Cent. Sci. 2017, 3 (10), 1070-1077. https://doi.org/10.1021/acscentsci.7b00222.

(43) Santa, T.; Al-Dirbashi, O. Y.; Fukushima, T. Derivatization Reagents in Liquid Chromatography/Electrospray Ionization Tandem Mass Spectrometry for Biomedical Analysis. Drug Discov. Ther. 2007, 1 (2), 108-118.

(44) Baskin, J. M.; Dehnert, K. W.; Laughlin, S. T.; Amacher, S. L.; Bertozzi, C. R. Visualizing Enveloping Layer Glycans during Zebrafish Early Embryogenesis. Proc. Natl. Acad. Sci. U.S.A 2010, 107 (23), 10360-10365. https://doi.org/10.1073/pnas.0912081107. Dehnert, K. W.; Baskin, J. M.; Laughlin, S. T.; Beahm, B. J.; Naidu, N. N.; Amacher, S. L.; Bertozzi, C. R. Imaging the Sialome during Zebrafish Development with Copper-Free Click Chemistry. Chembiochem 2012, 13 (3), 353-357. https://doi.org/10.1002/cbic.201100649.

Dehnert, K. W.; Beahm, B. J.; Huynh, T. T.; Baskin, J. M.; Laughlin, S. T.; Wang, W.; Wu, P.; Amacher, S. L.; Bertozzi, C. R. Metabolic Labeling of Fucosylated 
Glycans in Developing Zebrafish. ACS Chem. Biol. 2011, 6 (6), 547-552. https://doi.org/10.1021/cb100284d.

(47) Batt, A. R.; Zaro, B. W.; Navarro, M. X.; Pratt, M. R. Metabolic Chemical Reporters of Glycans Exhibit Cell-Type-Selective Metabolism and Glycoprotein Labeling. ChemBioChem 2017, 18 (13), 1177-1182.

https://doi.org/10.1002/cbic.201700020.

(48) Soriano Del Amo, D.; Wang, W.; Jiang, H.; Besanceney, C.; Yan, A. C.; Levy, M.; Liu, Y.; Marlow, F. L.; Wu, P. Biocompatible Copper(I) Catalysts for in Vivo Imaging of Glycans. J. Am. Chem. Soc. 2010, 132 (47), 16893-16899. https://doi.org/10.1021/ja106553e.

(49) Fishelson, L. A Comparative Study of Ridge-Mazes on Surface Epithelial CellMembranes of Fish Scales (Pisces, Teleostei). Zoomorphology 1984, 104 (4), 231238. https://doi.org/10.1007/BF00312036.

(50) Whitear, M. The Skin Surface of Bony Fishes. J Zool L. 1970, 160, 437-454.

(51) Hawkes, J. W. The Structure of Fish Skin - I. General Organization. Cell Tissue Res. 1974, 149 (2), 147-158. https://doi.org/10.1007/BF00222270.

(52) Le Guellec, D.; Morvan-Dubois, G.; Sire, J. Y. Skin Development in Bony Fish with Particular Emphasis on Collagen Deposition in the Dermis of the Zebrafish (Danio Rerio). Int. J. Dev. Biol. 2004, 48 (2-3), 217-231. https://doi.org/10.1387/ijdb.15272388.

(53) Saint-Amant, L.; Drapeau, P. Time Course of the Development of Motor Behaviors in the Zebrafish Embryo. J. Neurobiol. 1998, 37 (4), 622-632. https://doi.org/10.1002/(SICI)1097-4695(199812)37:4<622::AID- 
NEU10>3.0.CO;2-S. 


\section{FIGURES}

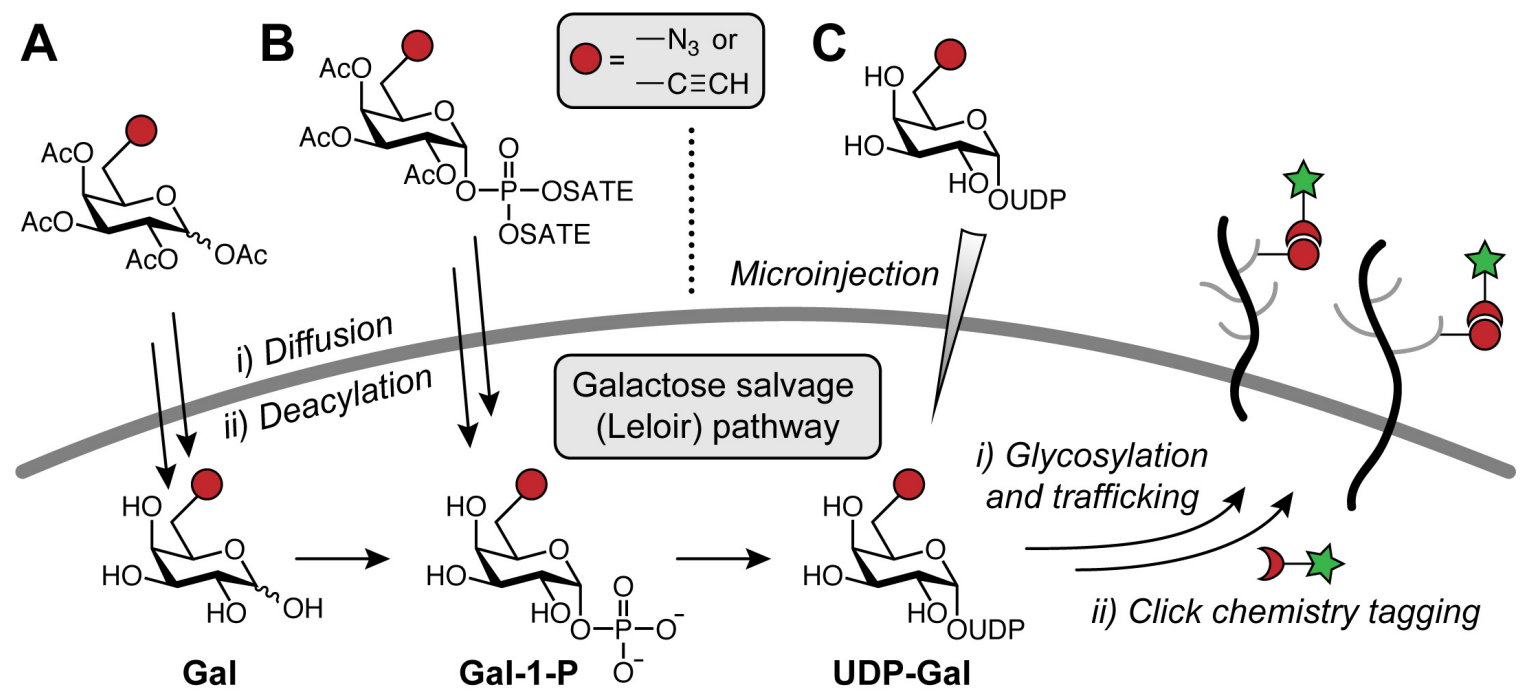

Figure 1. Strategy for using synthetic galactose analogs for metabolic labeling and imaging of cell-surface glycans. Galactose analogs were synthesized to intercept various steps of the galactose salvage pathway (Leloir pathway). Shown are analogs of galactose (A), galactose-1-phosphate (Gal-1-P) (B), and uridine diphosphate galactose (UDP-Gal) (C). Ac, acetyl; SATE, S-acetyl thioethyl; UDP, uridine diphosphate. Red ball indicates bioorthogonal tag (azide or acetylene), and crescent-star refers to bioorthogonal reaction partner to introduce epitope tag to enable visualization. See also Fig. S1 for structures of all analogs. 
A

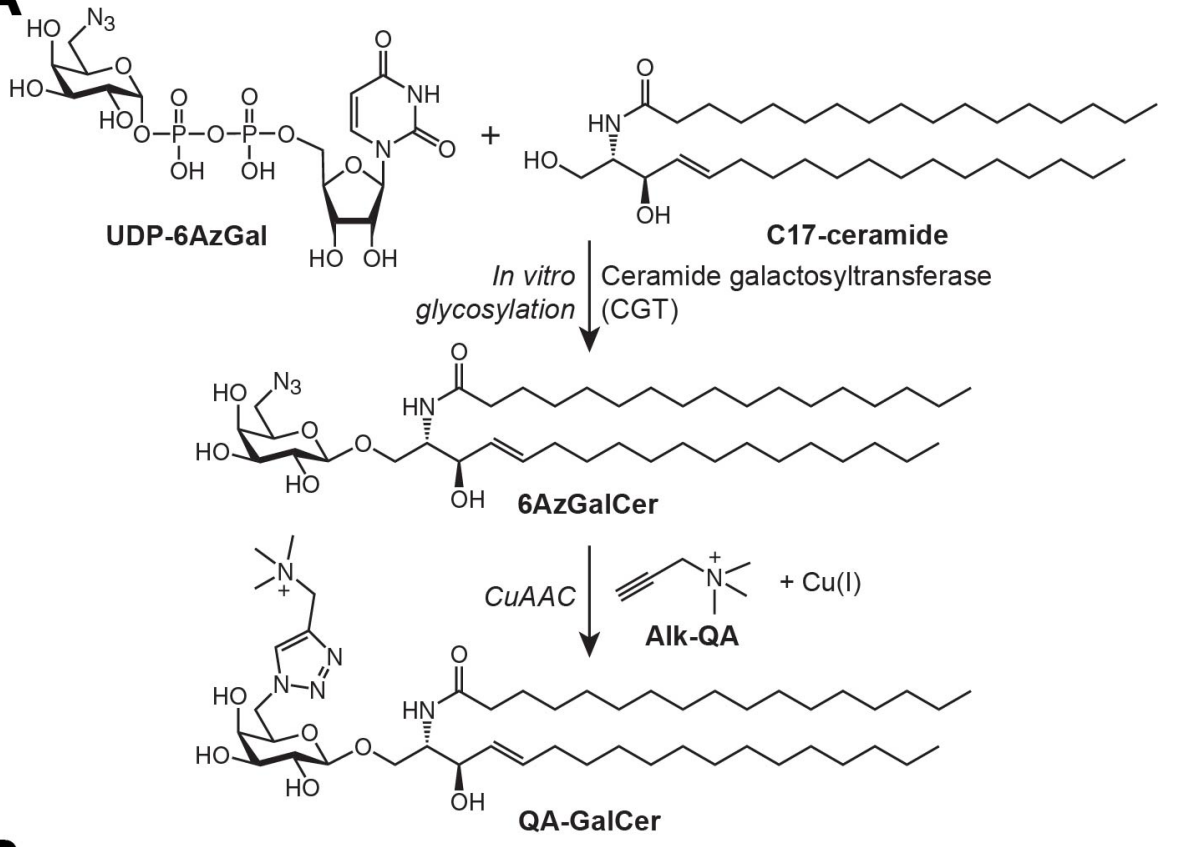

B

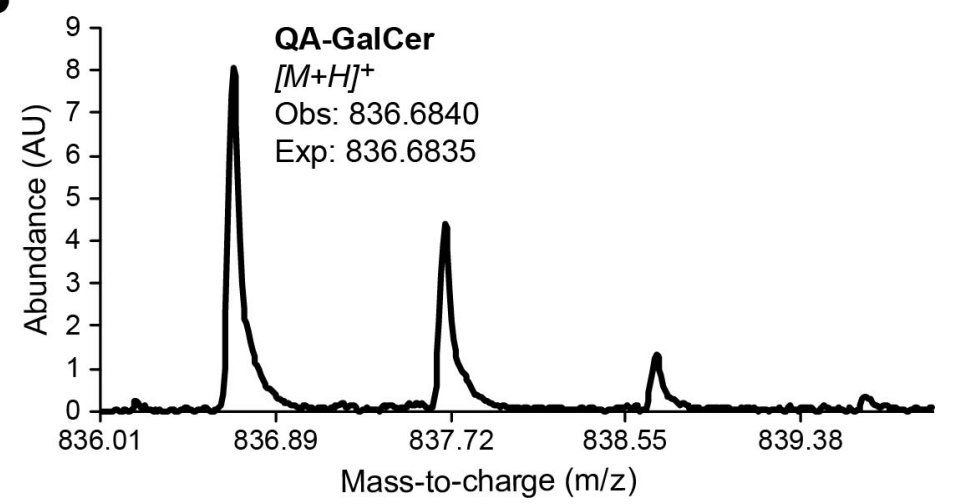

Figure 2. An azido analog of UDP-Gal is accepted by a galactosyltransferase in vitro.

UDP-6AzGal was incubated overnight at $37^{\circ} \mathrm{C}$ with $\mathrm{C} 17$-ceramide and lysates from $\mathrm{HEK}$ 293TN cells expressing CGT to generate the 6-azido analog of galactosylceramide (6AzGalCer). Following lipid extraction and CuAAC tagging with an alkynyl quaternary ammonium reagent (Alk-QA) to generate the quaternary ammonium lipid derivative (QAGalCer), analysis was performed by LC-MS (electrospray ionization-time of flight (ESI- 
TOF)). (A) Schematic of experimental design. (B) Mass spectrum showing identification of QA-GalCer, with observed and expected $\mathrm{m} / \mathrm{z}$ values indicated. 
A

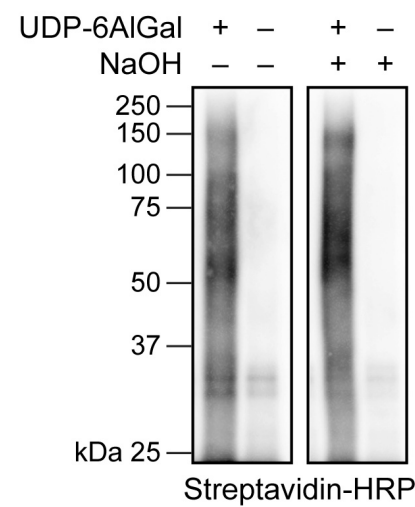

B

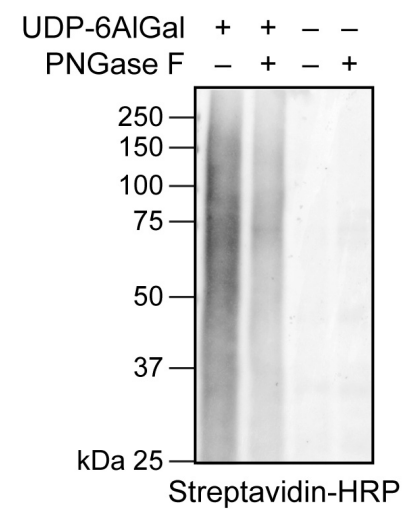

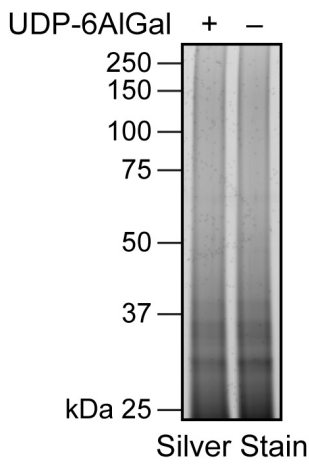

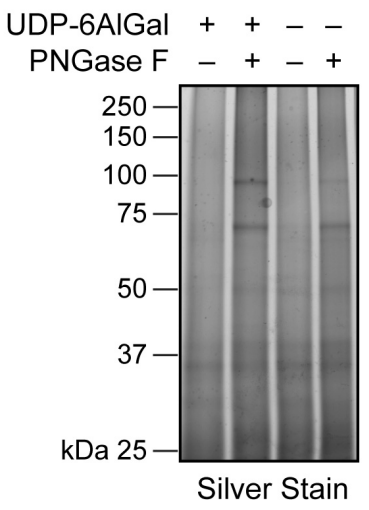

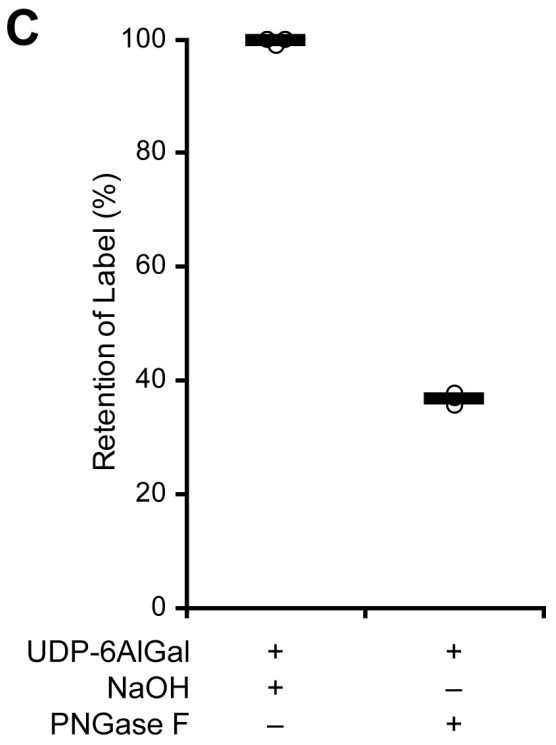

Figure 3. UDP-6AIGal labels N-linked glycans in developing zebrafish. Zebrafish embryos were microinjected with UDP-6AlGal or no sugar at the 1-cell stage and allowed to develop at $28{ }^{\circ} \mathrm{C}$ for $24 \mathrm{~h}$, followed by generation of protein lysates. (A) Lysates were reacted with azido-biotin by CuAAC and analyzed by SDS-PAGE and transfer to a PVDF membrane. At this point, membranes were incubated with or without $\mathrm{NaOH}(55 \mathrm{mM}, 40$ ${ }^{\circ} \mathrm{C}$ for $22 \mathrm{~h}$ ) to remove O-glycans, followed by Western blot with streptavidin-HRP or silver stain. (B) Lysates were incubated with PNGase F to remove N-glycans, followed by CuAAC tagging with azido-biotin and Western blot analysis with streptavidin-HRP or silver stain. (C) Quantification of Western blot data from (A) and (B), showing the 
retention of UDP-6AlGal-derived signal in the presence of $\mathrm{NaOH}$ (left) or PNGase $\mathrm{F}$ (right) treatment. Each experiment was performed three times (biological replicates) with three technical replicates for each experiment. 
$12 \mathrm{hpf}$
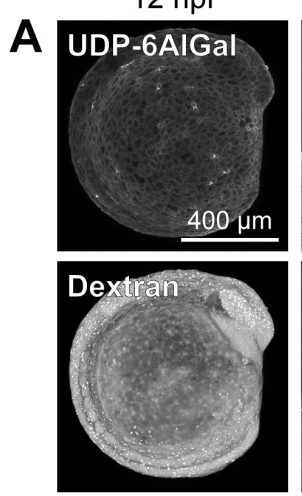

B No Sugar
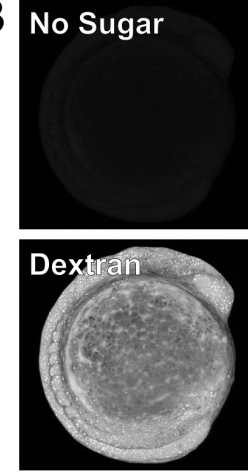

C
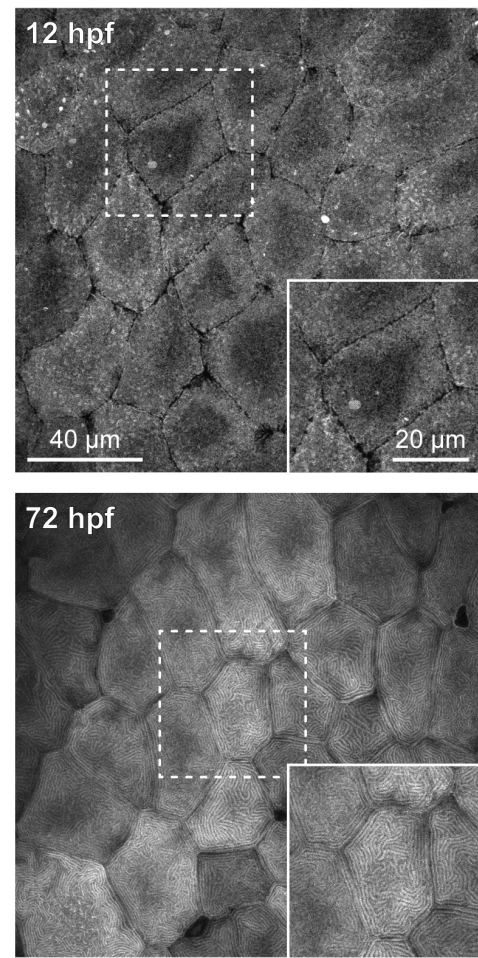

$48 \mathrm{hpf}$
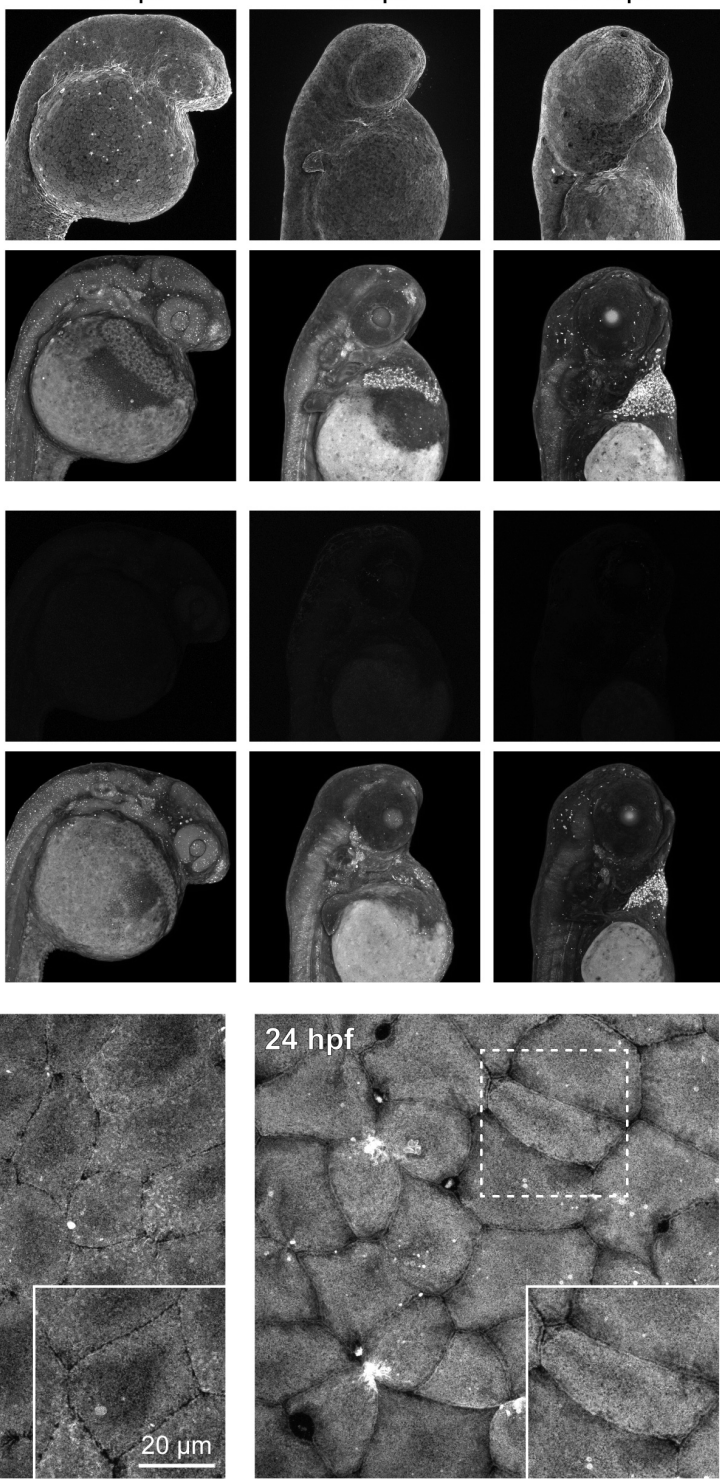

$96 \mathrm{hpf}$
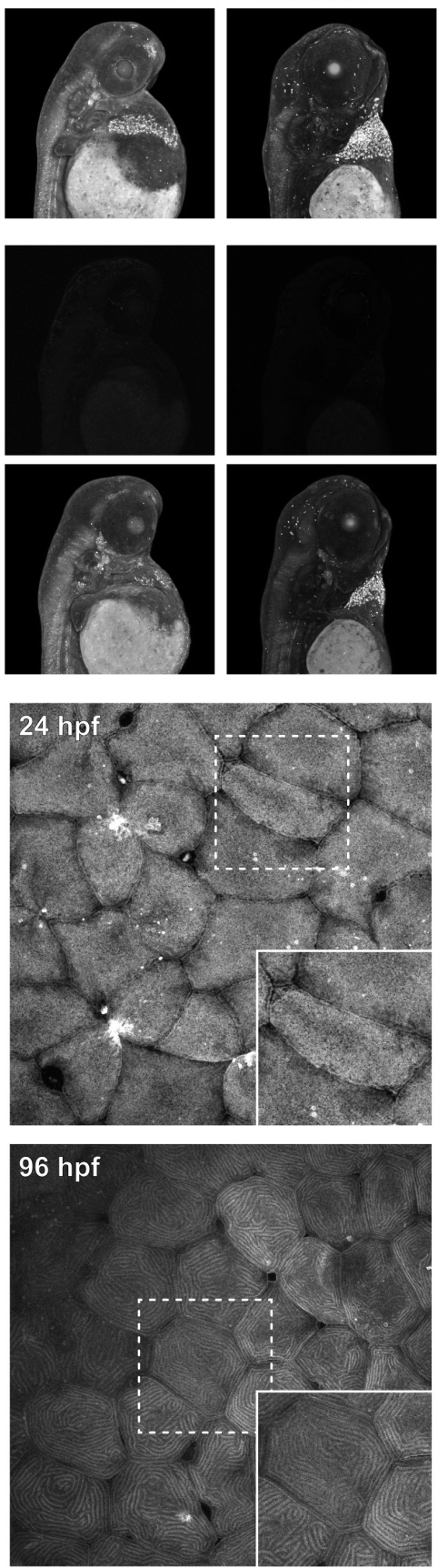

$96 \mathrm{hpf}$
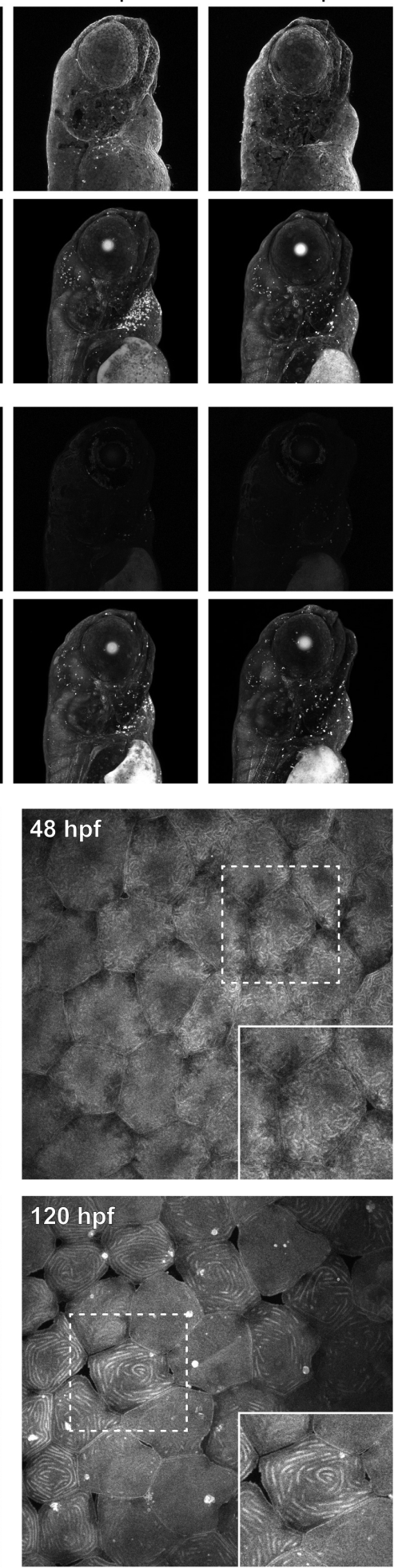
Figure 4. Imaging of glycosylation in developing zebrafish embryos and larvae using UDP-6AlGal and CuAAC. (A) Zebrafish embryos were microinjected with the red fluorescent tracer rhodamine-dextran and either UDP-6AlGal (A) or no sugar (B) at the 1cell stage and allowed to develop at $28{ }^{\circ} \mathrm{C}$ for the indicated amount of time up to $120 \mathrm{hpf}$ (hours post-fertilization). Live zebrafish were then tagged with azido-biotin via CuAAC, followed by labeling with streptavidin-Alexa Fluor 488, mounting in low-melt agarose, and imaging by confocal microscopy. Images in (C) show zoomed-in regions of enveloping layer cells on the surface of the embryo, above the yolk sac, highlighting intense UDP6AlGal-derived fluorescence in ridged structures; insets at bottom right show area in dashed line. 